\title{
Numerical investigation of the fluid structure acoustics interaction on a simplified car model
}

\author{
Katrin Nusser* and Stefan Becker \\ Friedrich-Alexander University Erlangen-Nürnberg, Institute of Process Machinery and Systems Engineering (iPAT), \\ Cauerstr. 4, 91058 Erlangen, Germany
}

Received 28 September 2020, Accepted 24 March 2021

\begin{abstract}
Part of vehicle interior noise is caused by the complex turbulent flow field behind the a-pillar and side mirror. It excites the structure of the side window, which radiates noise into the interior. Both aerodynamic pressure excitation and acoustic sound sources in the flow play an important role. In this work, the influence of both excitation mechanisms is investigated numerically in a hybrid simulation on a simplified car geometry. The generic model allows for an exact definition of boundary conditions and good reproducibility of simulation results. An incompressible Large-Eddy-Simulation (LES) of the flow is conducted, from which acoustic source terms within the flow field and transient fluid forces acting on the surface of the side window are extracted. This data is used in a coupled vibroacoustic and aeroacoustic simulation of the structure and passenger cabin of the vehicle. A finite element (FE) approach is used for the simulations and detailed modeling of the structure and the influence of interior absorption properties is emphasized. The computed excitation on the side window and the interior noise levels are successfully validated by using experimental data. The importance and contribution of both aerodynamic and acoustic pressure excitation to the interior sound level are determined.
\end{abstract}

\section{Introduction}

Reducing interior noise levels to improve driver and passenger comfort has gained increasing importance for researchers and acoustic engineers in the automotive industry. Since last-minute structural and design changes due to acoustic uncertainties are costly, it is necessary to be able to predict interior acoustics in an early development phase.

A significant contribution to the interior noise levels stems from the flow behind the side view mirror and the a-pillar [1]. The highly turbulent flow field in this area is characterized by velocity and pressure fluctuations with widely varying length scales. These fluctuations include sound sources present in the flow field and sound generated by the interaction of the flow with solid surfaces. A combination of acoustic pressure and aerodynamic wall-pressure fluctuations excite the flexible structure of the side window, leading to sound radiation into the interior. Both parts of the pressure differ in length scale, wave number, and energy content due to the disparity of scales. Therefore, they also cause different vibration characteristics of the structure, which will be examined in this work.

Different analytical, experimental and numerical methods have been applied to investigate the mechanisms of wind-induced noise radiation into the interior of a car.

*Corresponding author: katrin.nusser@fau.de
Many studies investigate isolated objects, like a simplified mirror model mounted on a flat plate or other wall-mounted obstacles. Numerical and experimental investigations were conducted for example by Siegert et al. [2], Becker et al. [3], Ask and Davidson [4] and Schäfer et al. [5]. Other research was done on the flow around simplified car models, for example by Krajnovic and Davidson [6] and Aljure et al. [7]. Many authors investigated the separation of aerodynamic wall-pressure fluctuations and acoustic pressure fluctuations on the side window and their influence on the interior sound level [8]. The experimental approaches rely for example on wavenumber filtering on the surface, as done by Arguillat et al. [1] and Van Herpe et al. [9]. In recent years, many numerical investigations of the problem were conducted, using different methods for sound calculation. Mendonca et al. [10] simulated the fully compressible flow around a simplified car model and used vibroacoustic and aeroacoustic methods to compute the sound pressure level in the interior. Similar investigations were also done on real car models, for example by Schell and Cotoni [11, 12], who used a hybrid approach consisting of an incompressible Computational Fluid Dynamics (CFD) simulation, frequency domain finite element computations and a statistical energy analysis (SEA) model for the interior cabin.

In the present work, the phenomenon of flow-induced noise is investigated numerically on the example of the SAE body, a simplified car model, with an added side view mirror. The workflow to assess this structural-acoustic 
coupling encompasses a large eddy simulation (LES) of the turbulent flow field around the car model, as well as aeroacoustic and vibroacoustic methods to predict the sound propagation outside and inside the car. For the simulations of structural dynamics and linear acoustics, the underlying basic equations are numerically solved in the frequency domain, using an finite element (FEM) approach. No statistical or integral methods are employed in this work. Between flow and acoustics, a hybrid simulation scheme is used. One part of the coupling approach is based on aeroacoustic sound sources calculated from the flow field at simulation time [13]. From these, the acoustic field on the outside of the car, the structural excitation of the side window due to loading by acoustic pressure, and the acoustic field on the inside of the SAE body are computed. The second coupling path investigates the excitation of the side window by aerodynamic pressure loading and the resulting radiation of sound into the interior. Both excitation paths are investigated separately. With this method, the influence of acoustic and aerodynamic pressure fluctuations on the excitation of the structure is determined and their respective contribution to the interior sound level is quantified.

All investigations focus on the frequency range between $100 \mathrm{~Hz}$ and $2500 \mathrm{~Hz}$. This frequency range is highly relevant since it dominates the overall SPL in the interior. A focus was placed on the close reproduction of the experimental setup, especially in terms of the vibration behavior of the side window and acoustical damping in the interior cabin. From previous works, extensive experimental results for this model are available, including surface pressure, microphone and laser-scanning vibrometer measurements $[14,15]$. Therefore, all major simulation steps are validated by experimental results.

This study shows that it is possible to accurately determine the SPL inside a car model at an early stage in the development process. The numerical methods used are solely based on the respective governing equations, without employing additional modeling approaches. This makes a high spatial and temporal resolution of the underlying physical phenomena possible. Additionally, it was discovered that a close characterization of the vibration properties of the structure and the acoustic damping in the interior cabin is crucial for accurately reproducing the interior sound field in the investigated frequency range. Since the influence of aerodynamic and aeroacoustic pressure fluctuations is investigated separately, it is also possible to quantify the individual contribution of each transfer paths on the resulting interior sound pressure level, which can be used to motivate constructional changes during the car development process.

This paper is organized as follows: we begin with a short description of the employed methods and then present the setup of the experiment, as well as CFD and FEM simulations. In the next section, we discuss the simulated flow field and compare the different contributions to the overall excitation of the side window. We also analyze the influence of the interior configuration of the model and compare the results to experimental data. Finally, we give concluding remarks concerning the results and the applicability of the employed methods.

\section{Methods}

A hybrid simulation approach [13] is used in this work to ensure that an optimal discretization and solution process can be selected for the underlying physical problem. The incompressible, turbulent flow field is solved by the CFD software FASTEST-3D [16], which is based on a finite volume approach. The aero-acoustic and vibroacoustic simulations are conducted by the FEM solver CFS $++[17]$. The latter simulations are executed in the frequency domain to incorporate impedance boundary conditions for the interior acoustic simulations and frequency-dependent mechanical damping of the structure. Therefore, data from the CFD simulation had to be transformed into the frequency domain using Fast-Fourier Transformation (FFT) before the coupling.

To reproduce the different characteristics of aerodynamic and aeroacoustic pressure fluctuations in the simulations and to investigate their influence on the interior sound levels, two separate excitation paths are investigated. The aerodynamic excitation path is based on pressure fluctuations caused by the incompressible turbulent flow field. These are exported from the CFD simulation and mapped onto the structure as pressure loading. The second excitation path by acoustic pressure is based on acoustic source terms in the turbulent flow. They are computed by the CFD solver, interpolated onto the CAA grid, and subsequently used to calculate the acoustic wave propagation outside the car. A two-way coupling approach is employed between the structure of the side window and the acoustic fields on the outside and inside of the SAE body. Both types of pressure loading lead to a vibration of the structure, which radiates sound into the interior of the car model. The resulting sound field in the cabin is dependent on its geometry and the amount of damping due to acoustic absorption materials.

The instantaneous flow field is computed based on the incompressible Navier-Stokes equations for Newtonian fluids. These equations describe the conservation of mass (1) and momentum (2):

$$
\begin{gathered}
\frac{\partial u_{i}}{\partial x_{i}}=0 \\
\rho_{0}\left(\frac{\partial u_{j}}{\partial t}+\frac{\partial\left(u_{i} u_{j}\right)}{\partial x_{i}}\right)=-\frac{\partial p}{\partial x_{j}}-\rho_{0} v \frac{\partial}{\partial x_{i}}\left(\frac{\partial u_{i}}{\partial x_{j}}+\frac{\partial u_{j}}{\partial x_{i}}\right) .
\end{gathered}
$$

The gravitational forces are neglected in this case. Here, $u_{i}$ denotes the velocity components in the coordinate directions $x_{i}, \rho_{0}$ is the constant density of the fluid, $v$ the kinematic viscosity, $p$ the flow pressure, and $t$ the time. To account for turbulence in the flow, a large eddy simulation (LES) with a Smagorinsky subgrid-scale model is used. It was shown in previous work $[18,19]$ that this turbulence modeling approach is best suited to capture the small turbulent structures which occur around the simplified car model.

In the acoustic regions, the propagation of sound is calculated based on the perturbed convective wave equation (PCWE) [20-22]: 


$$
\frac{1}{c_{0}^{2}} \frac{\mathrm{D}^{2} \Phi^{a}}{\mathrm{D} t^{2}}-\nabla \cdot \nabla \Phi^{a}=-\frac{1}{c_{0}^{2} \rho_{0}} \frac{\mathrm{D} p^{h}}{\mathrm{D} t} \quad \text { with } p^{a}=\rho_{0} \frac{\mathrm{D} \Phi^{a}}{\mathrm{D} t} .
$$

Here, $\Phi^{a}$ denotes the acoustic scalar potential, $p^{a}$ is the acoustic part of the pressure, $p^{h}$ is the incompressible, aerodynamic part of the pressure, and $\frac{\mathrm{D}}{\mathrm{D} t}=\frac{\partial}{\partial t}+\overline{u_{i}} \frac{\partial}{\partial x_{i}}$ is the substantial derivative. The acoustic source term constitutes the right-hand side of the equation and is calculated by evaluating the material derivative of the flow pressure in the incompressible CFD simulation. This formulation was chosen since it includes the separation of acoustic and aerodynamic pressure in the source region. By using the acoustic potential instead of the acoustic pressure formulation, only the first temporal derivative of the flow pressure has to be calculated for the source term, making it less sensitive to numerical noise in the flow field [20].

At the interfaces between mechanical and acoustic regions, the wall-normal component of the velocity of the solid $u_{\mathrm{i}}$ must be equal to the wall-normal component of the acoustic particle velocity $u_{i}^{a}$ :

$$
u_{i} n_{i}=u_{i}^{a} n_{i}
$$

Since acoustic and mechanical computation are conducted simultaneously, the influence of the exterior and interior acoustic pressure on the structural displacement is considered. The pressure load on the structure is included as mechanical stress $\sigma_{n}$ on the surface:

$$
\sigma_{n}=-n_{i} p^{a} .
$$

Both coupling conditions (4) and (5) have to be fulfilled at the interfaces between the acoustic and mechanical regions.

\section{Model setup}

The outer geometry of the simplified car model which is used in this work is identical to the SAE Type 4 body [23]. A side mirror with square cross section is attached to the model, as shown in Figure 1.

This generic, idealized model was designed to reduce the complexity of a real car and to focus on distinct regions of interest. It is well suited to investigate the propagation of flow-induced noise into the interior. The whole model is assumed to be acoustically insulated, such that noise can only be transmitted through the side window. With the experimental model, this was proven to be valid for frequencies larger than $100 \mathrm{~Hz}$ [14]. The area of the side window is therefore the main region of interest for the simulations. The general aerodynamic, but also the aero- and vibroacoustic properties of the physical model are reproduced in detail by the simulations to ensure that their results are comparable to experimental data.

\subsection{Experimental setup}

The experimental model was constructed using sandwich elements composed of wood and heavy layers to ensure sufficient sound insulation and to limit the influence of other sound transmission paths. Additionally, a springmass system was mounted on the interior walls of the model. It was proven by measurements that the acoustical insulation of the model is effective for frequencies above $100 \mathrm{~Hz}$ [14]. The dimensions of the side window were similar to real side windows used in passenger cars. It consisted of a glass plate with a thickness of $3 \mathrm{~mm}$ and was fixed to a wooden frame using silicone at every edge over a length of $30 \mathrm{~mm}$. The corners of the frame were also connected by silicone without any screws. The window lay swimming in a bed of sealing material and gaps between model and window were sealed and closed by tape. The mounting of the window into the body was designed to limit transmission of structure-borne sound.

Additionally, the interior absorption properties of a series production car were replicated in the model. To this end, different absorbing materials were mounted on the walls of the interior cabin. Porous absorbers with a thickness of $30 \mathrm{~mm}$ were attached to the interior walls and the roof. A thin felt carpet was placed on the floor. By using these absorbing materials, a reverberation time comparable to real cars was achieved [24].

The experimental measurements were performed under semi-free field conditions in the acoustic wind tunnel of the BMW group (see Fig. 2), which has a lower cut-off frequency of $50 \mathrm{~Hz}[25]$. Five $1 / 2^{\prime \prime}$ free-field condenser microphones were employed for the sound measurements in the interior cabin, e.g. at the position of the driver's ear facing the side window. To investigate the correlation of different pressure fluctuations at the surface, 39 piezo-resistive pressure transducers were evenly distributed on the side window. The sensors were inserted into drill holes in an aluminum plate and mounted flush with the surface.

Phase-resolved laser-scanning vibrometer measurements were carried out to identify the vibration modes of the side window. For this purpose, a single-point laser Doppler velocimeter was used together with a scanning vibrometer to observe the flexural modes of the glass plate at different frequencies. This measurement yielded amplitude and phase of the window vibration at each node of the measurement grid, which involved 1624 uniformly distributed measuring positions.

\subsection{CFD simulation}

The incompressible, three-dimensional flow field around the simplified car model was computed using the in-house CFD code FASTEST-3D [16]. It is based on a finite volume approach and uses block-structured, co-located meshes to solve the transient, incompressible Navier-Stokes equations.

The outer dimensions of the SAE body in the CFD simulation were identical to the physical model in Figure 1. To reduce the computational effort, a half body grid was used and symmetry in the span-wise direction assumed. It was proven in precursor simulations that this boundary condition has no major influence on the flow in the area of the side window. The computational domain measured $20.7 \mathrm{~m}$ in length, $5.8 \mathrm{~m}$ in width, and $7.0 \mathrm{~m}$ in height. 

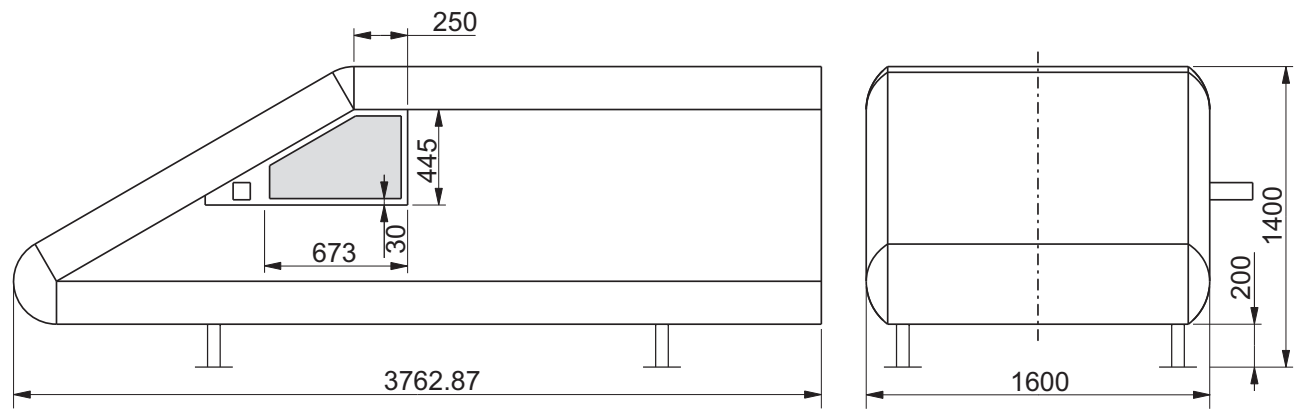

Figure 1. Schematic drawing of the dimensions of the simplified car model with units in mm.

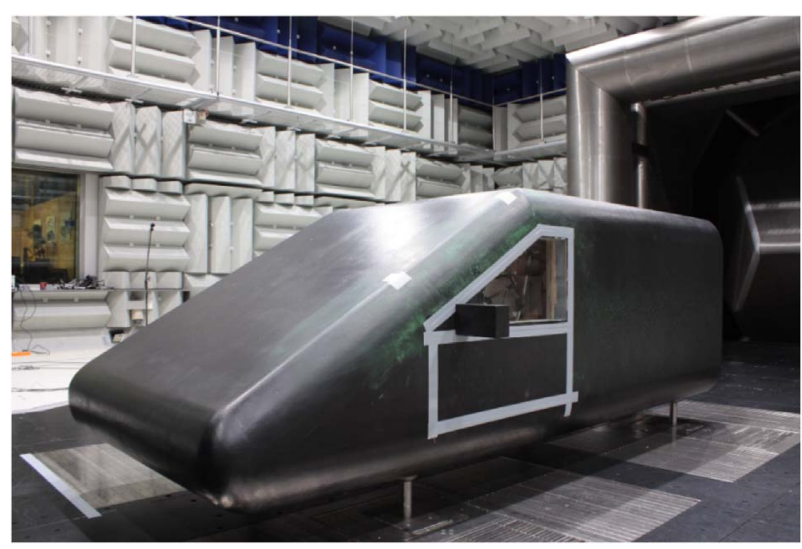

Figure 2. Simplified car model in acoustic wind tunnel [15].

The SAE body was placed $5.6 \mathrm{~m}$ upstream from the inlet. On the top, bottom and outer channel wall, a slip boundary condition was imposed. A convective outlet boundary condition was used to prevent pressure fluctuations due to reflections at the outlet. At the inlet, a free stream velocity of $u_{0}=100 \mathrm{~km} / \mathrm{h}$ was defined as a block profile with no additional synthetic turbulence. This simplification had no influence on the flow field behind the side mirror since the turbulence generated by the obstacle was much more pronounced. The flow was characterized by a Reynolds number of $1.47 \times 10^{5}$ based on the mirror height.

The flow domain was discretized using a blockstructured grid with a total size of $96.7 \mathrm{M}$ hexahedral control volumes. A fine near-wall resolution was achieved with the help of o-grids, which resulted in an average dimensionless distance of the first grid cell off the wall of $y^{+}<1$.

In the incompressible flow simulation with FASTEST3D, a large eddy simulation (LES) with a Smagorinsky subgrid-scale model was used in combination with a van Driest damping in the near-wall area. The Smagorinsky constant was set to 0.1. A central differencing scheme was employed for the discretization in space. Time advancement was realized via an implicit Crank-Nicolson scheme with a time step of $1.0 \times 10^{-6} \mathrm{~s}$, resulting in a Courant-FriedrichsLewy (CFL) number of 0.5. For velocity-pressure coupling, a predictor-corrector scheme was used.

The acoustic source terms were computed from the incompressible pressure $p^{h}$ during the CFD simulation and stored on the CFD grid. The computational grids of CFD and CAA simulations differed in dimensions and spatial resolution. Therefore, a conservative interpolation approach [26] is implemented to ensure an interpolation of the coupled quantities from the fine to the coarser grid without loss of information. To limit the influence of sound sources outside the region of interest and to prevent the creation of spurious sources, an additional blending function was applied to the source terms. Outside the vicinity of the side window, the source terms were assumed to be equal to zero.

\subsection{Aero-acoustic and vibro-acoustic model}

The coupled aeroacoustic and vibroacoustic simulation was realized by using the software $\mathrm{CFS}++[17]$, which is based on the finite element method. It simultaneously computed the sound propagation outside the model, the mechanical displacement of the side window due to acoustic or aerodynamic pressure loading, and the resulting sound radiation into the interior cabin.

The simulation domain is depicted in Figure 3. It includes three distinct regions: the outside of the SAE body, the interior cabin and the side window with coupling interfaces on both sides. The first two are acoustic regions, the side window is modeled as a flexible structure. The outside acoustic domain is surrounded by a perfectly matched layer (PML) [27] to damp the acoustic pressure to zero and to prevent reflections at the domain boundaries. For these simulations, a full model of the SAE body was used, since the interior acoustic field cannot be assumed to be symmetric. The outer surface of the SAE body and the floor of the domain were modeled as acoustically hard walls. The outer and inner surfaces of the side window constituted coupling interfaces between acoustic and mechanical regions. A conformal mesh was chosen at the interfaces, such that no additional interpolation was necessary between the different domains.

In the experimental setup, the side window consisted of a glass plate with a thickness of $3 \mathrm{~mm}$ mounted on a wooden frame that was inserted into the SAE body. This setup was optimized to limit the possible sound transmission paths to the free surface of the window. Since no screws were used for mounting, the boundary conditions for the simulation were not clearly defined. Therefore, an iterative 


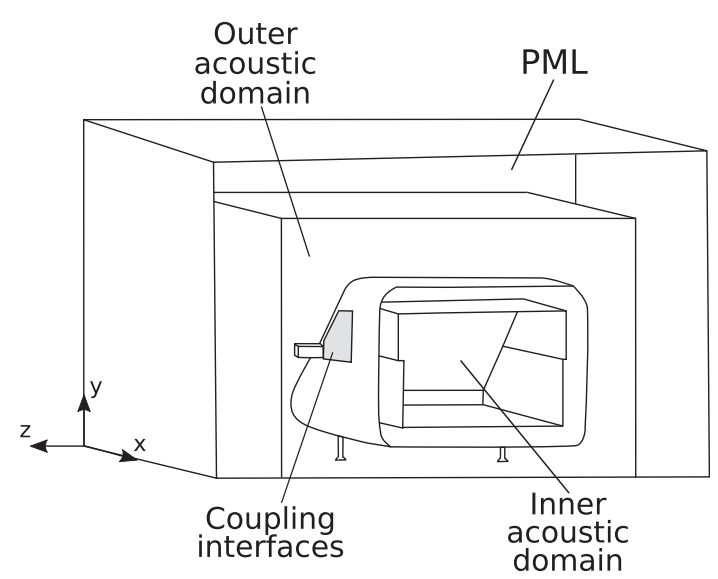

Figure 3. Schematic drawing of the simulation domain.

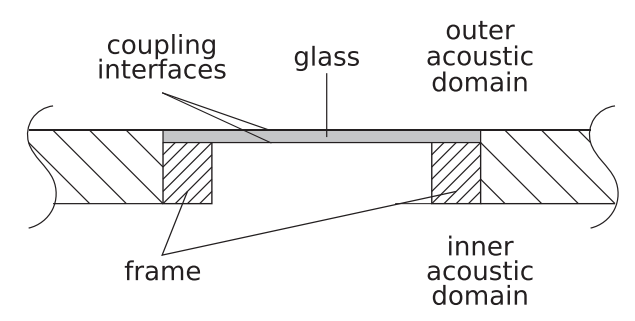

Figure 4. Schematic drawing of mechanical regions.

approach based on modal analysis was used to determine the relevant material properties and boundary conditions for the simulation setup. Correct reproduction of the vibration behavior of the side window was crucial, since it influences the interior sound pressure level to a high degree, especially in the investigated frequency range of $100 \mathrm{~Hz}$ to $3000 \mathrm{~Hz}$. These frequencies are located below the coincidence frequency of the side window of about $3900 \mathrm{~Hz}$, where the bending wavenumber of the structure is equal to the acoustic wavenumber.

The material properties of the glass plate were determined using the manufacturer's data. To reproduce the mounting of the window, the frame was modeled as a second mechanical region as depicted in Figure 4. Its material properties correspond to birch plywood. The material properties of both glass plate and frame are listed in Table 1. The inner part of the frame was fixed in all three coordinate directions. This modeling was verified by experimental and numerical eigenmode analysis. In the experimental setup, the glass window was exited by a white noise signal and the exerted force was measured. The resulting surface velocity of the glass was captured using a single-point vibrometer at 21 distributed measuring positions. The eigenmodes of the structure and their respective damping were identified through the frequency response function derived from the output response of the glass to the applied force. The simulated eigenmodes conform with the experimentally determined ones with a maximum deviation of $10 \%$. The damping effect in the frame was modeled with a Rayleigh damping model. The damping factors $\xi=\frac{\alpha}{2 \omega}+\frac{\beta \omega}{2}$, with $\alpha$
Table 1. Material properties of structural mechanics regions.

\begin{tabular}{lccc}
\hline Region & $\begin{array}{c}\text { Density } \\
\text { in } \mathrm{kg} / \mathrm{m}^{3}\end{array}$ & $\begin{array}{c}\text { Young's modulus } \\
\text { in Pa }\end{array}$ & $\begin{array}{c}\text { Poisson's } \\
\text { number }\end{array}$ \\
\hline Glass & 2500 & $70 \times 10^{9}$ & 0.22 \\
Frame & 900 & $85 \times 10^{5}$ & 0.45 \\
\hline
\end{tabular}

and $\beta$ denoting the mass and stiffness related damping respectively, were determined for different frequencies $\omega$ through experimental eigenmode analysis. Additional simulations showed, that using different damping factors for different frequencies only resulted in minor changes in the mechanical excitation. Therefore, a constant damping factor of $\xi=0.015$ was applied for all frequencies in the investigated frequency range.

The computational domain was discretized using tetrahedral cells with $3.0 \mathrm{M}$ nodes in total. The source region was meshed with a maximum element size of $15 \mathrm{~mm}$ and was refined to $4 \mathrm{~mm}$ in the direct vicinity of the side window (see Fig. 5). The inside of the cabin also used element sizes of $15 \mathrm{~mm}$. The surface of the side window consisted of triangles with a maximal side length of $4 \mathrm{~mm}$. The mechanical domain inside the side window used a conformal grid consisting of prisms with two elements in thickness direction. The frame on which the side window was mounted was also meshed conformally with 10 elements in thickness direction. For the discretization of the acoustic regions, first-order FE basis functions were used, whereas all mechanical regions employed second-order FE basis functions to avoid locking effects. Due to the grid sizes in the acoustic regions, acoustic waves with a frequency of $3000 \mathrm{~Hz}$ can be resolved with 7.6 elements per wavelength. At least six elements per wavelength are necessary to reduce the dispersion error to $15 \%$ [28]. The acoustic sound sources and also the aerodynamic forces on the side window were exported every $1 \times 10^{-5} \mathrm{~s}$. Acoustic waves with frequencies up to $5000 \mathrm{~Hz}$ can therefore be resolved with 20 points per period. A total physical time of $0.2 \mathrm{~s}$ was realized.

In the experiments, the interior cabin of the SAE body was lined with different types of absorbing material to achieve acoustical properties comparable to a series production car [14]. To emulate the acoustic characteristics of the interior in the simulation, all absorbing materials were characterized and their damping effect was included using normal impedance boundary conditions. The porous absorbers were characterized by flow resistance measurements and their impedance was calculated by using the DelanyBazley empirical model [29]. For the felt carpet, which covered the floor of the SAE body, complex impedance values of a similar carpet measured by Aretz [30] were used. The damping behavior of the spring-mass system was difficult to quantify due to its modular structure. Therefore, its absorption coefficient was determined by using Eyring's formula [31] with the known surface absorption values and the measured reverberation time of the interior cabin. The frequency-dependent impedance values of all damping materials are provided in the Supplementary Material. The model was validated by calculating the room transfer 


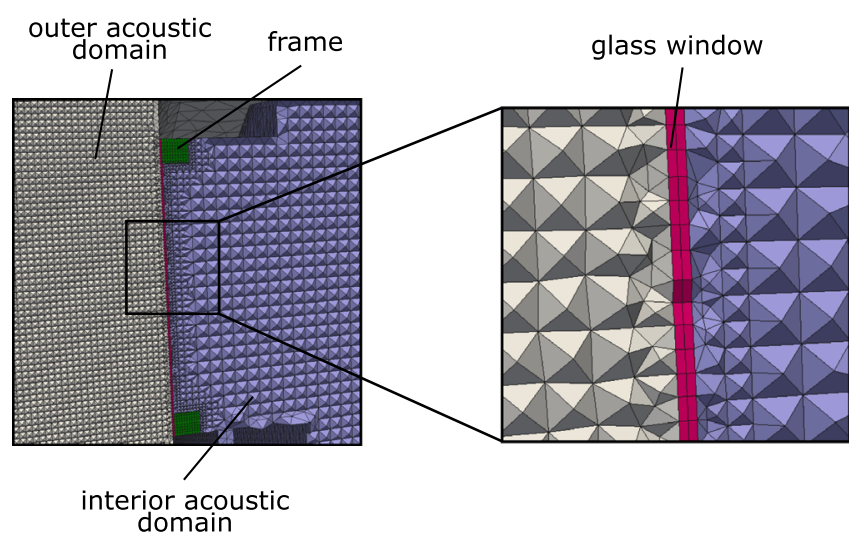

Figure 5. Section of acoustic grid in near window area.

function at different positions inside the SAE body and comparing them to experimental data. A good agreement was observed over the whole investigated frequency range.

\section{Results}

\subsection{Flow field}

To illustrate the instantaneous flow and the developing turbulent structures in the CFD simulation, the $Q$-criterion proposed by Hunt et al. [32] was used. Iso-surfaces of the second invariant of the velocity gradient tensor $Q$ are depicted in Figure 6. It shows a high degree of irregularity and a wide range of sizes of vortex structures generated by the flow behind mirror and a-pillar. The characteristic horseshoe vortex and the vortex shedding behind the obstacle can be observed. The vortex breakdown in the mirror wake is also clearly visible.

From measurements in an acoustic wind tunnel, surface pressure data at 39 positions on the side window was available. This data was compared to surface pressure from the CFD simulation at the same monitoring positions in the wake of the mirror model. The resulting wall pressure spectra are depicted in Figure 7.

At the position directly behind the side mirror (Fig. 7a), a good agreement between simulation and experiment can be observed up to a frequency of over $2000 \mathrm{~Hz}$. For higher frequencies, the resolution is limited by the spatial resolution of the CFD mesh, which acts as a low-pass filter for the smaller turbulent scales. At a measuring position further downstream (Fig. 7b), the frequency resolution is slightly decreased, since the surface mesh was coarsened further downstream from the side mirror. However, the overall resolution was sufficient for this application, since it focuses on the frequency range below $3000 \mathrm{~Hz}$. Influence of the periodic vortex shedding behind the mirror model is visible at both measuring locations as tonal peaks, especially below $200 \mathrm{~Hz}$. Approximating the wall-pressure fluctuations by an analytical model, e.g. the Corcos model, is therefore difficult in this frequency range. The Corcos model is based on the coherence properties of a turbulent boundary layer flow which are used to generate a synthetic

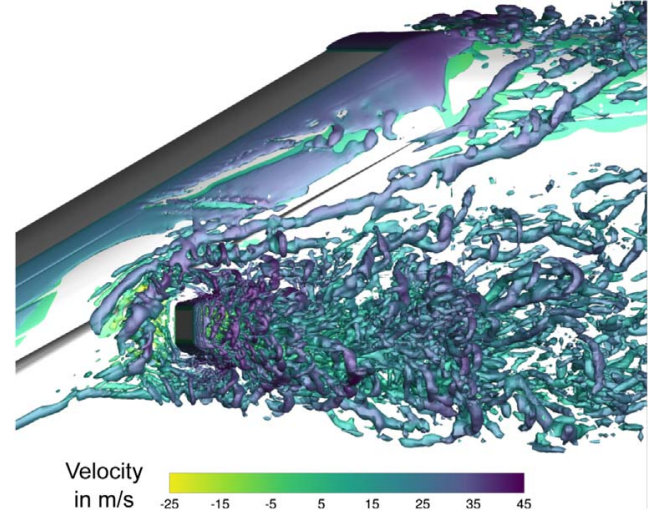

Figure 6. Isosurfaces of $Q$-criterium $\left(Q=2 \times 10^{5} 1 / \mathrm{s}^{2}\right)$ coloured by velocity magnitude.

pressure field. Therefore, it is only able to capture its broadband characteristics, but no tonal components. A detailed resolution of the turbulent structures in the CFD was therefore necessary in the low frequency range.

\subsection{Excitation mechanisms}

A hybrid approach was employed for the coupled acoustic and mechanical simulations. For the aeroacoustic excitation path, sound sources were computed at every time step during the incompressible CFD simulation. A formulation based on the total differential of the pressure (3) was used. Iso-surfaces of this source term transformed by FFT can be observed in Figure 8 at frequencies of $100 \mathrm{~Hz}$ and $500 \mathrm{~Hz}$. Only the upper $70 \%$ of the maximum amplitudes are displayed for clarity. At a frequency of $100 \mathrm{~Hz}$, the highest source strengths are located in the shear layers above and below the mirror. These areas are directly adjacent to the front part of the side window. At a frequency of $500 \mathrm{~Hz}$, the highest amplitudes of the source term are more evenly distributed and shifted to the direct vicinity of the mirror. Most are located in the area of the horseshoe vortex in front of the mirror and in the shear layers around it. However, one cannot deduce the acoustic pressure loading on the surface directly from the distribution of acoustic source terms since the acoustic propagation, including reflection and other phenomena, plays an important role.

The sound sources were interpolated conservatively onto the acoustic mesh and propagation of sound outside the car was computed. By using the previously described coupling procedure, the displacement of the side window and the resulting acoustic field in the interior was determined.

For the second, aerodynamic excitation path, the turbulent pressure load on the surface of the car model was computed during the CFD simulation. Figure 9 shows an exemplary distribution of the frequency-dependent surface pressure at $150 \mathrm{~Hz}$ and $500 \mathrm{~Hz}$.

Similar characteristics as in case of the acoustic sources are visible here. The size of the turbulent structures decreases with increasing frequency. At a frequency of $150 \mathrm{~Hz}$, the areas with the highest pressure amplitude are located in the front part of the window, directly behind 


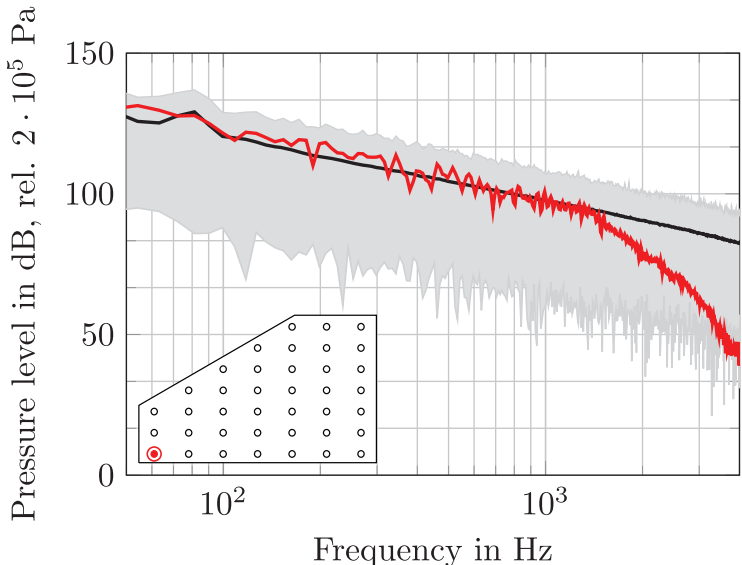

(a) Pos. 1

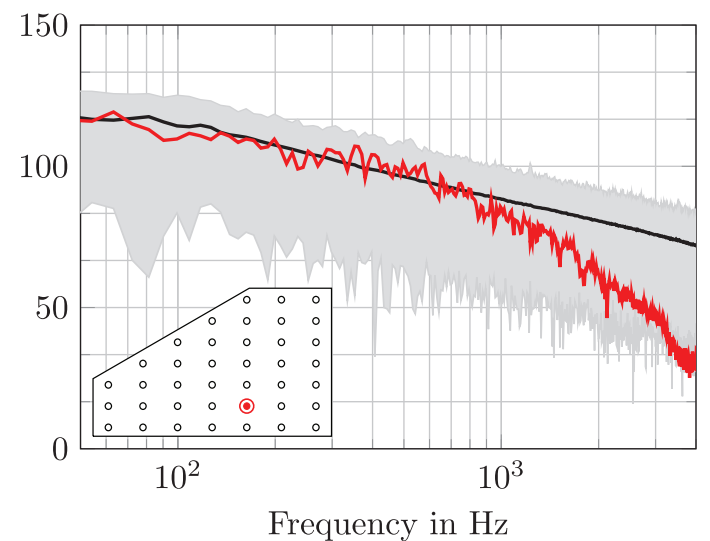

(b) Pos. 20

experiment, $0.2 \mathrm{~s}-$ experiment - simulation

Figure 7. Frequency spectra of the wall-pressure fluctuations in the wake of the mirror model.

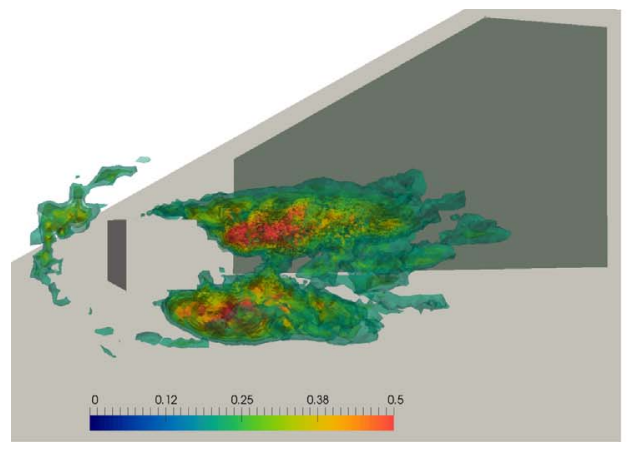

(a) $100 \mathrm{~Hz}$

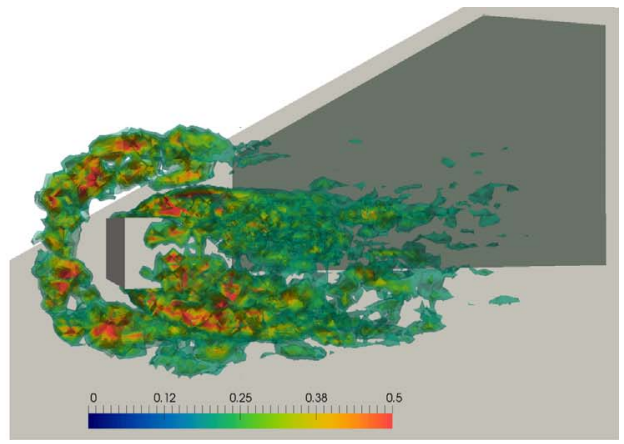

(b) $500 \mathrm{~Hz}$

Figure 8. Iso-surfaces of acoustic source term (3) calculated by CFD simulation at different frequencies.

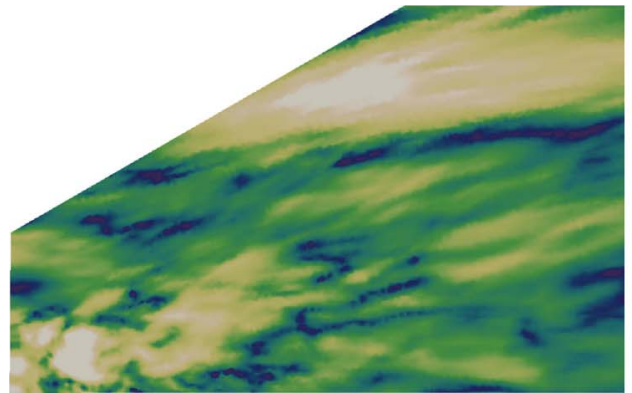

$\mathrm{p}^{\prime}$ in $\mathrm{dB}$ rel. $2 \mathrm{e}-5 \mathrm{~Pa}$

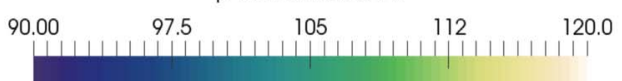

(a) $150 \mathrm{~Hz}$

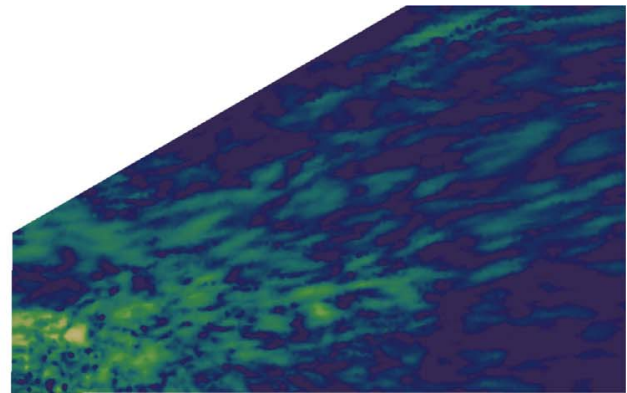

$\mathrm{p}^{\prime}$ in $\mathrm{dB}$ rel. $2 e-5 \mathrm{~Pa}$

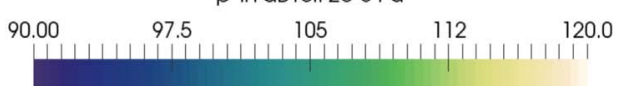

(b) $500 \mathrm{~Hz}$

Figure 9. Aerodynamic pressure distribution on surface from CFD simulation at different frequencies.

the mirror model. Additionally, the vortex structures generated by the flow around the a-pillar influence the upper part of the window. For higher frequencies, the areas of high pressure loading are smaller and more concentrated in the wake of the mirror. No direct influence of the a-pillar vortex is visible here.
From these investigations of the different excitation mechanisms, it becomes clear that they are highly frequency dependent. Especially at low frequencies, no uniform broadband characteristics can be observed. The vortex shedding and the generated large turbulent flow structures have a significant impact in this frequency range. 


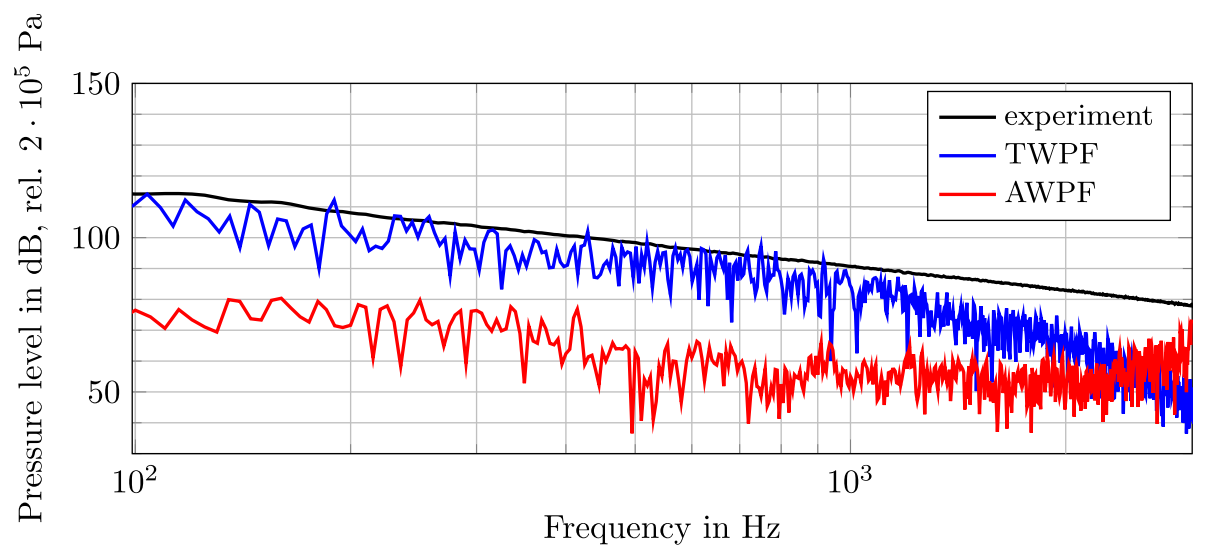

Figure 10. Comparison of acoustic (AWPF) and turbulent wall pressure fluctuations (TWPF) with experimental data at center of side window.

The resulting acoustic and turbulent wall pressure fluctuations are visualized at a position in the center of the side window in Figure 10. The acoustic pressure fluctuations (AWPF) were calculated by solving the equations for acoustic wave propagation on the outside of the SAE body. The turbulent wall pressure fluctuations (TWPF) were exported directly from the incompressible flow field. Both parts of the pressure are compared to experimentally determined data.

These measured values show a characteristic slope from $100 \mathrm{~Hz}$, which is also reproduced by the TWPF in the simulation. Both are also very similar in amplitude. In comparison, the AWPF show a much lower amplitude and therefore energy content as the TWPF. An offset of $20 \mathrm{~dB}$ to $30 \mathrm{~dB}$, depending on the frequency, can be observed. The AWPF cannot be measured directly, but they can be determined from experimental data, e.g. by a wavenumber decomposition which makes it possible to separate both parts of the pressure. Using this approach, similar offsets between acoustic and aerodynamic pressure were reported in previous experimental investigations [11, 14, 33].

Both AWPF and TWPF exhibit a similar slope over the frequency range between $200 \mathrm{~Hz}$ and $500 \mathrm{~Hz}$. Above $500 \mathrm{~Hz}$, AWPF levels remain nearly constant up to $2500 \mathrm{~Hz}$. Above this frequency, both types of pressure fluctuations are influenced by the CFD resolution. The rise in AWPF level for these frequencies is caused by an increase in numerical noise, whereas the drop in TWPF is caused by filtering effects due to the limited spatial resolution of the CFD simulation. Frequencies below $100 \mathrm{~Hz}$ will not be included in the following discussions since the validity of the experimental measurements is limited below this value due to the lower cut-off frequency of the wind tunnel and the absorbers. Additionally, the extend of the simulated outer acoustic region cannot accommodate acoustic waves with larger wavelengths.

\subsection{Vibroacoustics}

For both excitation paths, the acoustic and aerodynamic pressure fluctuations act as a pressure loading on the structure, causing it to vibrate. In the simulations, the resulting displacement of each element of the structure is easily determined. These were compared to experimental vibrometer data for validation. The measurements were conducted in the aeroacoustic wind tunnel at a free stream velocity of $150 \mathrm{~km} / \mathrm{h}$ [15]. The simulation results, however, are only available for an inlet velocity of $100 \mathrm{~km} / \mathrm{h}$. Thus, the displacement amplitudes cannot be compared directly to the measurements, but the general characteristics can be investigated qualitatively.

A comparison of simulated displacement and experimental data is depicted at two different frequencies in Figure 11. These frequencies coincide with the first and second eigenmodes of the structure. The shape of the first eigenmode at $120 \mathrm{~Hz}$ is captured well by the simulations. The maximum amplitude of the simulated displacement is about $30 \%$ lower than the measured one, which can be explained by the differences in free stream velocity. The resulting displacement amplitudes in case of acoustic and aerodynamic excitation are similar, although the exciting pressure amplitudes differ by about $30 \mathrm{~dB}$ (see Fig. 10). This suggests an efficient excitation of the structure in case of AWPF loading. Additionally, the in-phase vibration of the side window can also be observed below the first eigenmode, between the first and second, and the second and third eigenmode (not pictured). The general shape is similar to the first eigenmode, with no node lines visible. For TWPF excitation, this vibration mode only occurs below and at the first eigenmode.

The frequency of the second simulated eigenmode deviates slightly from the experimentally determined frequency of $190 \mathrm{~Hz}$. This can be attributed to small discrepancies in the interior configuration and the mounting of the window which both influence the experimental measurements. These influences can also lead to differences in the mode shape, which can be observed here in the location of the maximum displacement amplitude. However, both mode shapes are similar overall. The displacement amplitude of the simulation with acoustic excitation is $10 \%$ smaller than for aerodynamic excitation.

As a next step, reasons for the different vibration behavior are investigated in more detail. To this end, the relation between surface pressure loading and displacement 


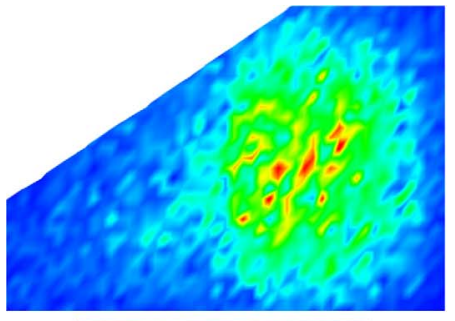

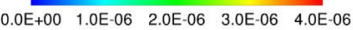

(a) Experiment, $120 \mathrm{~Hz}$

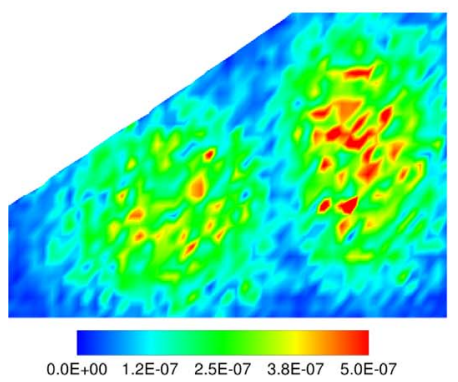

(d) Experiment, $190 \mathrm{~Hz}$
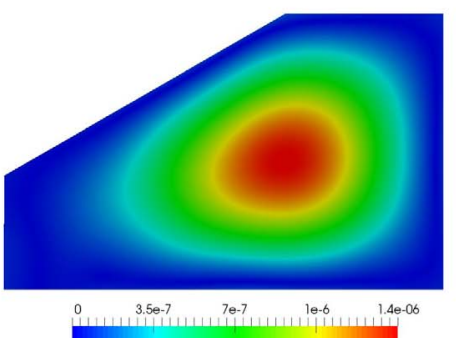

(b) TWPF, $120 \mathrm{~Hz}$

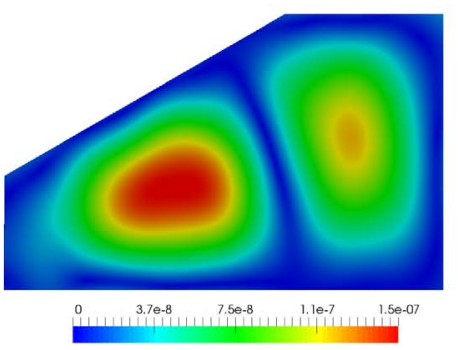

(e) TWPF, $200 \mathrm{~Hz}$

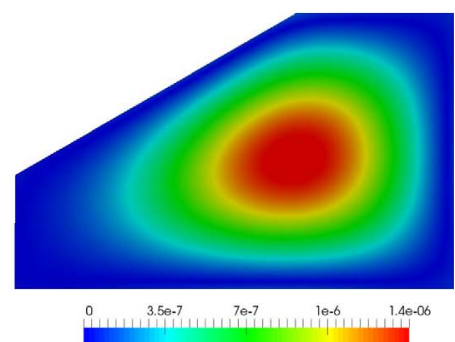

(c) AWPF, $120 \mathrm{~Hz}$

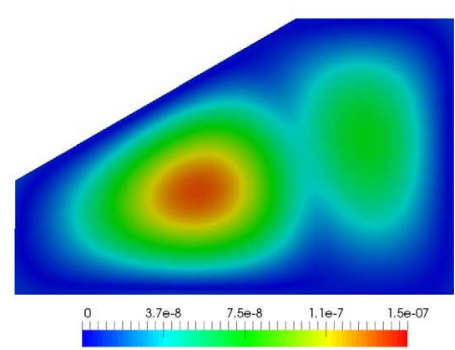

(f) $\mathrm{AWPF}, 200 \mathrm{~Hz}$

Figure 11. Comparison of displacement of side window in $\mathrm{m}$ from vibrometer measurements $(150 \mathrm{~km} / \mathrm{h})[15]$ and simulation $(100 \mathrm{~km} / \mathrm{h})$ with aerodynamic and acoustic pressure excitation.

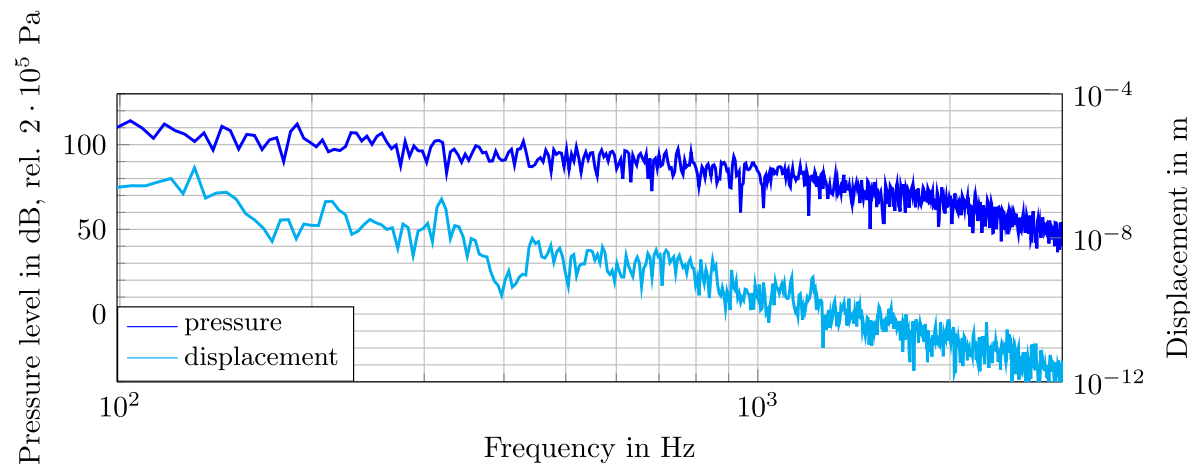

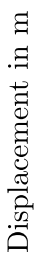

(a) aerodynamic excitation

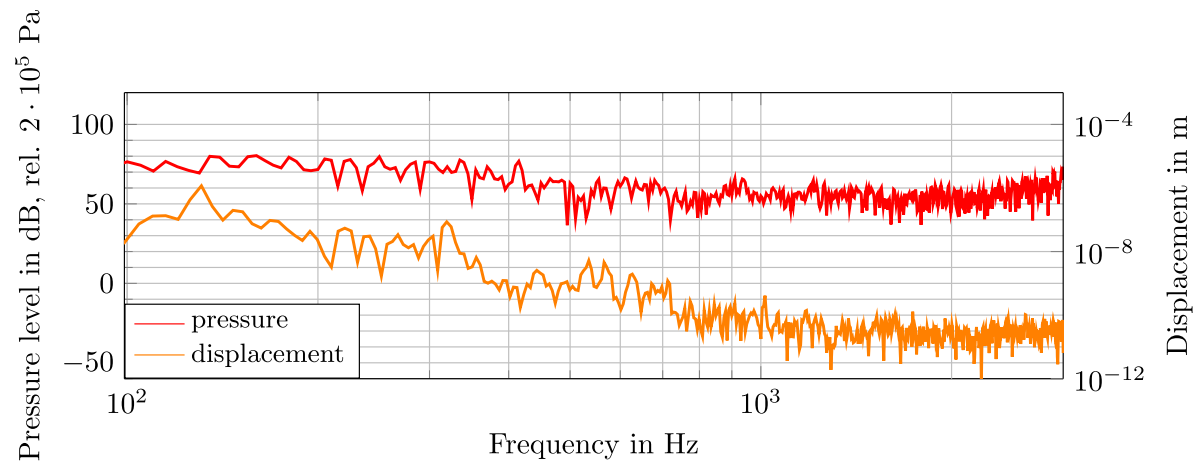

(b) acoustic excitation

Figure 12. Relation of wall pressure fluctuations and structural displacement at the center of the side window.

of the structure is displayed in Figure 12a for aerodynamic excitation and in Figure 12b for acoustic excitation. Pressure and displacement amplitude are visualized at a position in the center of the side window. When investigating the correlation between aerodynamic wall pressure fluctuations and the resulting structural displacement in Figure 12a, both curves show a similar characteristic. This suggests a connection between the energy content of the pressure 
excitation and the resulting displacement of the structure. The latter is elevated at the location of the eigenfrequencies of the structure, which are clearly visible for lower frequencies. The wavenumber of the aerodynamic pressure fluctuations is very different from the bending wavenumber of the structure, which impedes an efficient coupling of both below the coincidence frequency. Only a resonant excitation of the eigenmodes of the structure by TWPF of the same frequency is possible here. However, below the coincidence frequency, the sound radiation efficiency of resonant modes is small, since they are located in the acoustic short circuit region. This is partially compensated by the energy content of the TWPF, which is about three orders of magnitude higher than in case of AWPF.

For an excitation by acoustic pressure, the displacement amplitude is in the same range as in case of TWPF excitation. It is also elevated at the location of the eigenfrequencies of the structure and shows a more broadband characteristic for higher frequencies due to the increased modal density. The structural displacement curve and the pressure amplitude show no direct relation. The latter remains almost constant over the whole frequency range, whereas the former is highest in the low-frequency range up to $350 \mathrm{~Hz}$. These high displacement amplitudes are mainly attributed to the in-phase vibration of the structure in this region. For frequencies below the coincidence frequency of the structure $\left(f_{\mathrm{c}}=3900 \mathrm{~Hz}\right)$ and excitation by a diffuse acoustic field, radiated sound can be solely attributed to the excitation of non-resonant modes [34, 35]. This can be attributed to a similarity between the acoustic wavenumber of the exciting AWPF and the eigenvalues of the structure, which makes an efficient coupling between both possible [36]. In this case, sound is radiated at the eigenfrequency of the structural mode with a high radiation efficiency of $\sigma \approx 1$. This was also verified for the experimental measurements [15], where eigenmodes at frequencies below $400 \mathrm{~Hz}$ were excited by AWPF with a frequency of $1000 \mathrm{~Hz}$. Thus, it is concluded that an efficient, non-resonant excitation of the structure by AWPF can be observed. This becomes especially obvious in the relation between the energy content of the excitation and the resulting structural displacement.

\subsection{Interior acoustics}

Since the experiments were conducted using absorbing materials inside the cabin, it was important to determine the influence of this absorption on the simulated interior sound pressure level. For this purpose, an acoustic simulation of the interior of the car was conducted, using the experimentally determined normal velocity of the flexible structure as a boundary condition. It was measured from the inside of the SAE body by a laser-scanning vibrometer to rule out any influence of the exterior flow field. By using measured data as excitation in the simulations, it was possible to validate the interior modeling without influences of the previous simulation steps.

The resulting interior sound pressure level of simulations with and without absorbing boundary conditions inside the cabin are compared to reference values from experiments in Figure 13. The measurement position is located at the driver's ear at a distance of $0.25 \mathrm{~m}$ from the side window, a height of $0.7 \mathrm{~m}$ above the floor, and $0.1 \mathrm{~m}$ from the rear edge of the window. Due to the limited resolution of the experimental data, only frequencies up to $1000 \mathrm{~Hz}$ will be investigated in the following. Above this frequency, the structural displacement amplitude approaches zero and the signal becomes increasingly noisy.

Without damping materials, all interior surfaces are treated as fully reflecting. This leads to an overestimation of the SPL over the whole frequency range. The largest peaks can be observed at the locations of the interior cavity modes. At these frequencies, the SPL is up to $30 \mathrm{~dB}$ higher than in the damped case. The discrepancies in amplitude height can be attributed to differences in the energy content of the modes and to relations between mode shape and measurement position. When comparing the acoustically hard with the fully damped case, a large attenuation of these cavity resonances becomes apparent. The amplitude is decreased by up to $30 \mathrm{~dB}$ and shifted to lower frequencies. Not all modes are influenced to the same degree due to the uneven distribution of the absorbing material. For some modes, e.g. at frequencies of $160 \mathrm{~Hz}$ and $190 \mathrm{~Hz}$, the SPL is still overestimated by $10 \mathrm{~dB}$ in the damped case compared to the experimental data. The porous absorbers with the highest damping effect are located at the lower half of the side and rear walls and on the roof of the SAE body. The absorption coefficient of the other surfaces is smaller. Due to these inhomogeneities, it is difficult to ascribe a certain damping behavior to a specific material configuration. However, it becomes apparent that is important to include a sufficient amount of absorbing material to reduce the effect of cabin modes. In the fully damped case, the simulation is able to closely reproduce the measured interior SPL for frequencies up to $600 \mathrm{~Hz}$. For higher frequencies, the simulation data becomes influenced by the increased noise of the excitation data. In conclusion, the detailed replication of the damping properties of the experimental model in the simulation is very important to correctly recreate the characteristics of the interior sound field.

As a final step, the resulting acoustic field in the interior of the SAE body was determined. As previously discussed, the characteristic of the radiated sound is dependent on the type of excitation. To this end, the resulting sound pressure level at the position of the driver's ear is plotted separately in Figure 14a for excitation by acoustic and aerodynamic pressure fluctuations. The simulated SPL is compared to experimental data, which contains both excitation pressure contributions. When analyzing the contributions of both excitation mechanisms, it becomes clear that none of them can be neglected in the simulation of the interior sound field. Both have a large impact in different frequency ranges. The contribution of the acoustic pressure excitation is largest in the low-frequency range below $300 \mathrm{~Hz}$ and above $1500 \mathrm{~Hz}$. The former can be attributed to the nonresonant excitation of the structure and the resulting inphase vibration of the side window, which leads to high sound radiation. In the upper frequency range, the sound 


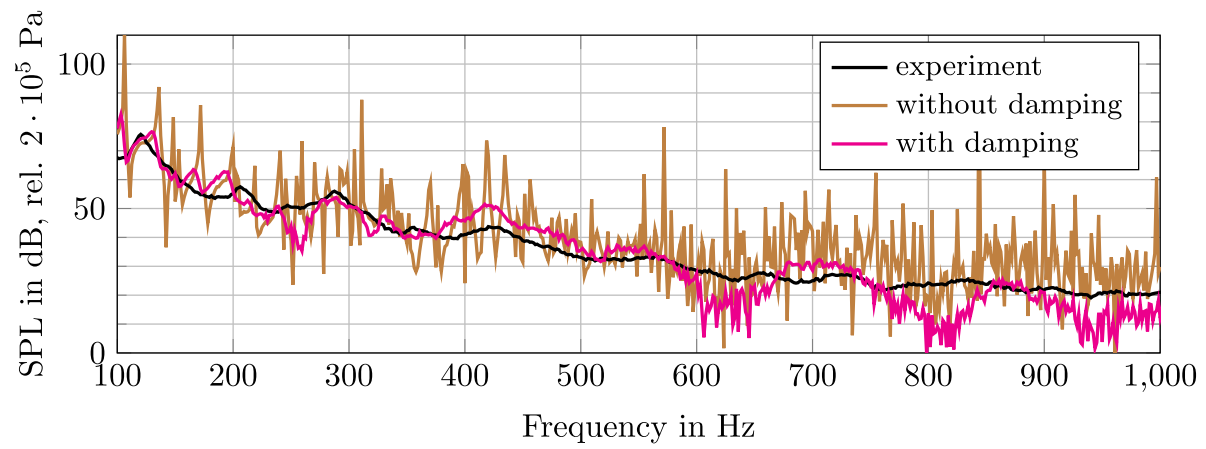

Figure 13. Comparison of different acoustic boundary conditions in the inside of the cabin.

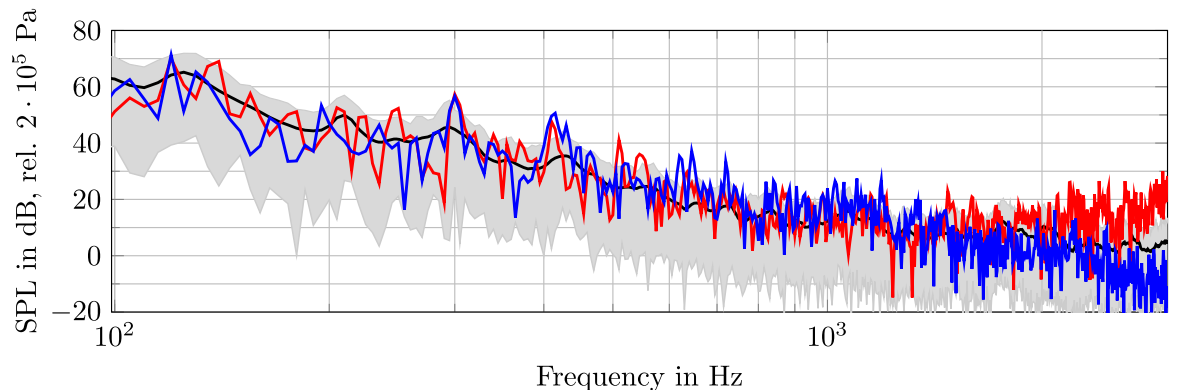

(a)

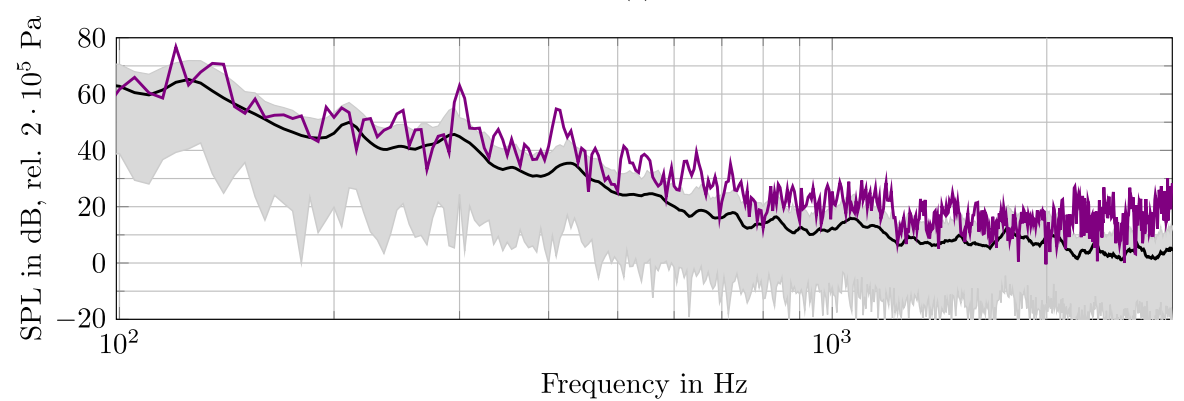

(b)

- experiment experiment, $0.2 \mathrm{~s}$ - acoustic excitation — aerodynamic excitation

- combined aerodynamic + acoustic excitation

Figure 14. Contribution of acoustic and aerodynamic pressure excitation to overall simulated SPL at driver's ear and comparison to experimental data.

radiation efficiency increases due to the increasing similarity of acoustic and structural wavenumber when approaching the coincidence frequency.

In Figure 14b, the contributions by both parts of the pressure are combined. Up to a frequency of $2200 \mathrm{~Hz}$, simulated and experimental data are in good agreement. Above this frequency, the limited spatial resolution of the CFD simulation influences the results. In the low-frequency range up to $500 \mathrm{~Hz}$, the SPL is mainly influenced by the resonance frequencies of the side window, which correspond to the peaks in SPL. The location of these eigenfrequencies is well reproduced by the simulation. This underlines the importance of realistic modeling of the bearing and material properties of the structure in the low-frequency range. Since damping materials were included in the simulation, the eigenmodes of the interior cavity only have a minor influence on the acoustic field.

\section{Conclusion}

In this work, the first-principles computation of windinduced noise and its transmission through the side window of a simplified car model without the use of empirical correlations, transfer functions, and experimental calibrations was achieved. For this, three distinct physical problems had to be solved: aerodynamics for sound generation, vibration for sound transmission through the side window and acoustics for the propagation of sound in the vehicle cabin and also outside of the car from the acoustic sound sources 
in the flow. These investigations were carried out on simplified car geometry with clearly defined properties and boundary conditions which can serve as a generic test case. All steps of the simulation workflow were validated against experimental data in the frequency range between $100 \mathrm{~Hz}$ and $2500 \mathrm{~Hz}$.

The flow-induced sound field inside a simplified car model was computed using a hybrid simulation approach. The turbulent flow around the SAE body was simulated by an incompressible LES approach. Acoustic source terms based on the perturbed acoustic wave equation and aerodynamic pressure loading on the structure were evaluated during the runtime of the CFD simulation. A fully coupled structural and acoustic simulation based on an FE approach was conducted by using aerodynamic and acoustic pressure loading as an excitation of the structure. In both cases, the propagation of sound inside the cabin was simulated and the resulting sound pressure level at the driver's ear was analyzed. The investigated low-frequency range makes a close reproduction of the experimental setup necessary, especially in terms of the vibration behavior of the side window and the acoustical damping in the interior cabin. The eigenmodes of the side window have a significant impact on the interior SPL for frequencies below $500 \mathrm{~Hz}$. Acoustic damping materials in the cabin were characterized and included in the simulation model. This was necessary to attenuate the cavity resonances, which have a large influence on the sound field in the undamped case.

A close reproduction of the sound pressure level in the interior of the SAE body was achieved by the simulation up to a frequency of $2200 \mathrm{~Hz}$. Additionally, it was possible to analyze the different excitation mechanisms of both acoustic and aerodynamic pressure fluctuations separately and to quantify their contribution to the total interior SPL. It was concluded, that both excitation mechanisms play an integral role in determining the total interior SPL due to their different excitation characteristics.

\section{Acknowledgments}

The authors gratefully acknowledge the compute resources and support provided by the Erlangen Regional Computing Center (RRZE).

\section{Supplementary material}

Supplementary material is available for this paper at https://acta-acustica.edpsciences.org/10.1051/aacus/ $2021014 / \mathrm{olm}$. It includes the frequency-dependent impedance values for the damping materials in the interior cabin:

impedance-absorber.txt: frequency-dependent real and imaginary part of impedance of absorber.

impedance-floor.txt: frequency-dependent real and imaginary part of impedance of felt carpet.

impedance-spring-mass.txt: frequency-dependent real and imaginary part of impedance of spring-mass system.

\section{References}

1. B. Arguillat, D. Ricot, G. Robert, C. Bailly: Measurements of the wavenumber-frequency spectrum of wall pressure fluctuations under turbulent flows, in 11th AIAA/CEAS Aeroacoustics Conference. American Institute of Aeronautics and Astronautics, May 2005.

2. R. Siegert, V. Schwarz, J. Reichenberger: Numerical simulation of aeroacoustic sound generated by generic bodies placed on a plate: Part-II, prediction of radiated sound pressure, in Proceedings of the 5th AIAA/CEAS Aeroacoustics Conference, 1999. American Institute of Aeronautics and Astronautics, May, 1999.

3. S. Becker, C. Hahn, M. Kaltenbacher, R. Lerch: Flowinduced sound of wall-mounted cylinders with different geometries. AIAA Journal 46 (2008) 2265-2281.

4. J. Ask, L. Davidson: A numerical investigation of the flow past a generic side mirror and its impact on sound generation. Journal of Fluids Engineering 131 (2009) 061102.

5. F. Schäfer, S. Müller, T. Uffinger, S. Becker, J. Grabinger, M. Kaltenbacher: Fluid-structure-acoustic interaction of the flow past a thin flexible structure. AIAA Journal 48 (2010) 738-748.

6. S. Krajnovic, L. Davidson: Flow around a simplified car, part 1: Large eddy simulation. Journal of Fluids Engineering 127 (2005) 907-918.

7. D. Aljure, I. Rodriguez, O. Lehmkhul, R. Borrell, A. Oliva: Flow and turbulent structures around simplified car models. Conference on Modelling Fluid Flow (2012) 247-254.

8. M. Cabrol, Y. Detandt, M. Hartmann, A. Mutzke: A comparison between the effects of turbulent and acoustic wall pressure fluctuations inside a car, in 18th AIAA/CEAS Aeroacoustics Conference (33rd AIAA Aeroacoustics Conference). American Institute of Aeronautics and Astronautics, June 2012, pp. 2012-2202.

9. F. Van Herpe, D. D'Udekem, J. Jacqmot, R. Kouzaiha: Vibro-acoustic simulation of side windows and windshield excited by realistic CFD turbulent flows including car cavity, in SAE Technical Paper Series. SAE International, June, 2012, pp. 01-1521.

10. F.G. Mendonca, T. Connelly, S. Bonthu, P. Shorter: CAEbased prediction of aero-vibro-acoustic interior noise transmission for a simple test vehicle. Tech. Rep. (2014) 1-2.

11. A. Schell, V. Cotoni: Prediction of interior noise in a sedan due to exterior flow. SAE International Journal of Passenger Cars - Mechanical Systems 8 (2015) 1090-1096.

12. A. Schell, V. Cotoni: Flow induced interior noise prediction of a passenger car, in INTER-NOISE and NOISE-CON Congress and Conference Proceedings, Vol. 254. Institute of Noise Control Engineering, 2017, pp. 1-10.

13. S. Schoder, M. Kaltenbacher: Hybrid aeroacoustic computations: State of art and new achievements. Journal of Theoretical and Computational Acoustics 27, 4 (2019) 1950020.

14. S. Müller, S. Becker, C. Gabriel, R. Lerch, F. Ullrich: Flowinduced input of sound to the interior of a simplified car model depending on various setup parameters, in 19th AIAA/CEAS Aeroacoustics Conference. American Institute of Aeronautics and Astronautics, May 2013.

15. S. Müller: Fluid-Struktur-Akustik-Kopplung bei der Überströmung dünner Platten. $\mathrm{PhD}$ thesis, Friedrich-AlexanderUniversität Erlangen-Nürnberg (FAU), April 2015.

16. F. Durst, M. Schäfer: A parallel block-structured multigrid method for the prediction of incompressible flows. International Journal for Numerical Methods in Fluids 22 (1996) 549-565. 
17. M. Kaltenbacher: Advanced simulation tool for the design of sensors and actuators. Procedia Engineering 5 (2010) $597-600$.

18. S. Becker, K. Nusser, M. Oswald: Aero-vibro-acoustic wind noise-simulation based on the flow around a car, in SAE Technical Paper Series. SAE International, June 2016.

19. K. Nusser, S. Müller, C. Scheit, M. Oswald, S. Becker: Large eddy simulation of the flow around a simplified car model, in Direct and Large-Eddy Simulation X. Springer International Publishing, 2017, pp. 243-249.

20. A. Hüppe, J. Grabinger, M. Kaltenbacher, A. Reppenhagen, G. Dutzler, W. Kühnel: A non-conforming finite element method for computational aeroacoustics in rotating systems, in 20th AIAA/CEAS Aeroacoustics Conference. American Institute of Aeronautics and Astronautics, 2014, p. 2739.

21. M. Kaltenbacher, A. Hüppe, A. Reppenhagen, F. Zenger, S. Becker: Computational aeroacoustics for rotating systems with application to an axial fan. AIAA Journal 55 (2017) $3831-3838$

22. S. Schoder, C. Junger, M. Kaltenbacher: Computational aeroacoustics of the eaa benchmark case of an axial fan. Acta Acustica 4, 5 (2020) 22.

23. N. Lindener: Aerodynamic Testing of Road Vehicles in Open Jet Wind Tunnels, vol. SP-1465 of SP (Society of Automotive Engineers). Society of Automotive Engineers, Warrendale, PA, 1999.

24. S. Müller, S. Becker, C. Gabriel, F. Ullrich: Influence of the flow-induced structure-borne noise on the interior acoustics of a simplified car model, in European Conference on Noise Control, Pragu, 2012, pp. 10-13.

25. M. Islam, F. Decker, M. Hartmann, A. Jaeger, T. Lemke, J. Ocker, V. Schwarz, F. Ullrich, A. Schroeder, A. Heider: Investigations of sunroof buffeting in an idealised generic vehicle model - Part I: Experimental results, in 14th AIAA/ CEAS Aeroacoustics Conference (29th AIAA Aeroacoustics Conference), American Institute of Aeronautics and Astronautics, May 2008, p. 2900.
26. M. Kaltenbacher, M. Escobar, S. Becker, I. Ali: Computational aeroacoustics based on lighthill's acoustic analogy, in Computational Acoustics of Noise Propagation in FluidsFinite and Boundary Element Methods. Springer, 2008, pp. $115-142$.

27. J.-P. Berenger: A perfectly matched layer for the absorption of electromagnetic waves. Journal of Computational Physics 1142 (1994) 185-200.

28. S. Marburg: Discretization Requirements: How many Elements per Wavelength are Necessary? Springer Berlin Heidelberg, Berlin, Heidelberg, 2008, pp. 309-332.

29. M.E. Delany, E.N. Bazley: Acoustical properties of fibrous absorbent materials. Applied Acoustics 3 (1970) 105-116.

30. M. Aretz, Combined wave and ray based room acoustic simulations of small roomss, vol. 12 of Aachener Beiträge zur technischen Akustiks. Logos Verlag Berlin GmbH, 2012.

31. C.F. Eyring: Reverberation time in "dead" rooms. The Journal of the Acoustical Society of America 1 (1930) 217-241.

32. J. Hunt, A. Wray, P. Moin: Eddies, stream, and convergence zones in turbulent flows. Center for Turbulence Research Report CTR-S88, 1988, pp. 193-208.

33. R.G. Dejong, T.S. Bharj, J.J. Lee: Vehicle wind noise analysis using a SEA model with measured source levels, in SAE Technical Paper Series. SAE International, April2001.

34. P. Bremner, J. Wilby: Aero-vibro-acoustics: Problem statement and methods for simulation-based design solution, in 8th AIAA/CEAS Aeroacoustics Conference. American Institute of Aeronautics and Astronautics, June 2002.

35. M. Hartmann, J. Ocker, T. Lemke, A. Mutzke, V. Schwarz, H. Tokuno, R. Toppinga, P. Unterlechner, G. Wickern: Wind noise caused by the side-mirror and a-pillar of a generic vehicle model, in 18th AIAA/CEAS Aeroacoustics Conference. American Institute of Aeronautics and Astronautics, June 2012.

36. W.K. Blake: Mechanics of flow-induced sound and vibrations. Academic Press, 1986.

Cite this article as: Nusser K \& Becker S. 2021. Numerical investigation of the fluid structure acoustics interaction on a simplified car model. Acta Acustica, 5, 22. 